\title{
Recognizing Human Actions from Still Images with Latent Poses
}

\author{
Weilong Yang, Yang Wang, and Greg Mori \\ School of Computing Science \\ Simon Fraser University \\ Burnaby, BC, Canada \\ wya16@sfu.ca, ywang12@cs.sfu.ca, mori@cs.sfu.ca
}

\begin{abstract}
We consider the problem of recognizing human actions from still images. We propose a novel approach that treats the pose of the person in the image as latent variables that will help with recognition. Different from other work that learns separate systems for pose estimation and action recognition, then combines them in an ad-hoc fashion, our system is trained in an integrated fashion that jointly considers poses and actions. Our learning objective is designed to directly exploit the pose information for action recognition. Our experimental results demonstrate that by inferring the latent poses, we can improve the final action recognition results.
\end{abstract}

\section{Introduction}

Consider the two images shown in Fig. 1(left). Even though only still images are given, we as humans can still perceive the actions (walking, playing golf) conveyed by those images. The primary goal of this work is to recognize actions from still images. In still images, the information about the action label of an image mainly comes from the pose, i.e. the configuration of body parts, of the person in the image. However, not all body parts are equally important for differentiating various actions. Consider the poses shown in Fig. 1(middle). The configurations of torso, head and legs are quite similar for both walking and playing golf. The main difference for these two actions in terms of the pose is the configuration of the arms. For example, "playing golf" seems to have very distinctive V-shaped arms, while "walking" seems to have two arms hanging on the side. A standard pose estimator tries to find the correct locations of all the body parts. The novelty of our work is that we do not need to correctly infer complete pose configuration in order to do action recognition. In the example of "walking" versus "playing golf", as long as we get correct locations of the arms, we can correctly recognize the action, even if the locations of other body parts are incorrect. The challenge is

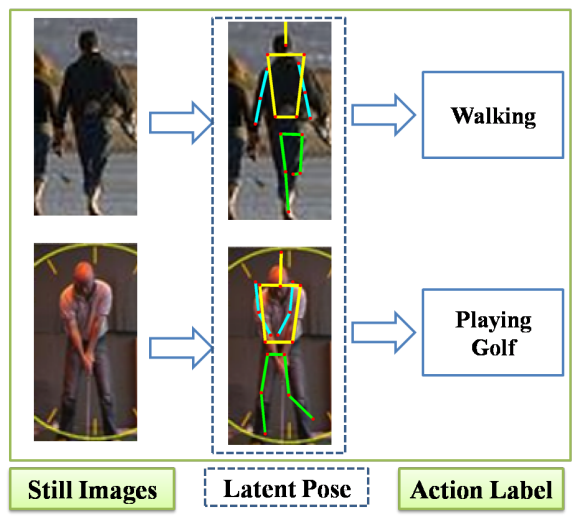

Figure 1. Illustration of our proposed approach. Our goal is to infer the action label of a still image. We treat the pose of the person in the image as "latent variables" in our system. The "pose" is learned in a way that is directly tied to action classification.

how to learn a system that is aware of the importance of different body parts, so it can focus on the arms when trying to differentiate between "walking" and "playing golf". We introduce a novel model that jointly learns poses and actions in a principled framework.

Human action recognition is an extremely important and active research area in computer vision, due to its wide range of applications, e.g. surveillance, entertainment, human-computer interaction, image and video search, etc. Space constraints do not allow an extensive review of the field, but a comprehensive survey is available in [9]. Most of the work in this field focuses on recognizing actions from videos $[13,15,18]$ using motion cues, and a significant amount of progress has been made in the past few years. Action recognition from still images, on the other hand, has not been widely studied. We believe analyzing actions from still images is important. Progress made here can be directly applied to videos. There are also applications that directly require understanding still images of human actions, e.g. news/sports image retrieval and analysis.

Not surprisingly, recognizing human actions from still images is considerably more challenging than video se- 
quences. In videos, the motion cue provides a rich source of information for differentiating various actions. But in still images, the only information we can rely on is the shape (or the pose) of the person in an image. Previous work mainly focuses on building good representations for shapes and poses of people in images. Wang et al. [20] cluster different human poses using distances calculated from deformable shape matching. Thurau and Hlaváč [19] represent actions using histograms of pose primitives computed by nonnegative matrix factorization. Ikizler et al. [10] recognize actions using a descriptor based on histograms of oriented rectangles. Ikizler-Cinbis et al. [11] learn actions from web images using HOG descriptors [3]. A limitation of these approaches is that they all assume an image representation based on global templates, i.e. an image is represented by a feature descriptor extracted from the whole image. This representation has been made popular due to its success in pedestrian detection, in particular the work on histogram of oriented gradient (HOG) by Dalal and Triggs [3]. This representation might be appropriate for pedestrian detection, since most pedestrians are upright. So it might be helpful to represent all the pedestrians using a global template. But when it comes to action recognition, global templates are not flexible enough to represent the huge amount of variations for an action. For example, consider the images of the "playing golf" action in Fig. 4. It is hard to imagine that a single global template can capture all the pose variations of this action. Recently, Felzenszwalb et al. [6] show that partbased representations can better capture the pose variations of an object, hence outperform global template representations. In this paper, we operationalize on the same intuition and demonstrate that part-based representations are useful for action recognition in still images as well. A major difference of our work from [6] is that we have ground-truth labeling of the pose on the training data, i.e. our "parts" are semantically meaningful.

Another important goal of this paper is to bridge the gap between human action recognition and human pose estimation. Those are two closely related research problems. If we can reliably estimate the pose of a person, we can use this information to recognize the action. However, in the literature, they are typically touted as two separate research problems and there has been only very little work on combining them together. There is some work on trying to combine these two problems in a cascade way, e.g. by building an action recognition system on top of the output of a pose estimation system. For example, Ramanan and Forsyth [17] annotate and synthesize human actions in 3D by track people in 2D and match the track to an annotated motion capture dataset. Their work uses videos rather than still images, but the general idea is similar. Ferrari et al. [8] retrieve TV shots containing a particular 2D human pose by first estimating the human pose, then searching shots based

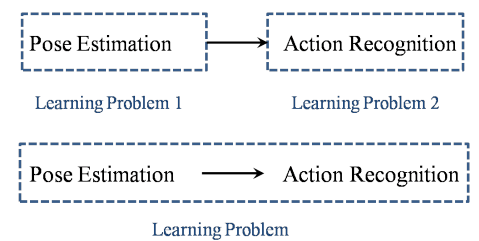

Figure 2. Difference between previous work and ours. (Top) Previous work typically approaches pose estimation and action recognition as two separate problems, and uses the output of the former as the input to the latter. (Bottom) We treat pose estimation and action recognition as an single problem, and learn everything in an integrated framework.

on a feature vector extracted from the pose. But it has been difficult to establish the value of pose estimation for action recognition in this cascade manner, mainly because pose estimation is still a largely unsolved problem. It is questionable whether the output of any pose estimation algorithm is reliable enough to be directly used for action recognition.

In this paper, we propose a novel way of combining action recognition and pose estimation together to achieve the end goal of action recognition. Our work is different from previous work in two perspectives. First, instead of representing the human pose as the configuration of kinematic body parts [16], e.g. upper-limb, lower-limb, head, etc., we choose to use an exemplar-based pose representation, "poselet". This notation of "poselet" is first proposed in [2] and used to denote a set of patches with similar 3D pose configuration. In this paper, for the purpose of action recognition, we further restrict those patches not only to have similar configuration, but also from the same action class. Second, as illustrated by the diagram in Fig. 2 (top), previous work typically treats pose estimation and action recognition as two separate learning problems, and uses the output of a pose estimation algorithm as the input of an action recognition system $[8,10]$. As pointed out earlier, the problem with this approach is that the output of the pose estimation is typically not reliable. Instead, as illustrated by the diagram in Fig. 2 (bottom), we treat pose estimation and action recognition as two components of a single learning problem, and jointly learn the whole system in an integrated manner. But our learning objective is designed in a way that allows pose information to help action classification.

The high-level idea of our proposed approach can be seen from Fig. 1. Our goal is to infer the action label of a still image. We treat the pose of the person as intermediate information useful for recognizing the action. But instead of trying to infer the pose correctly using a pose estimation algorithm, we treat the pose as latent variables in the whole system. Compared with previous work on exploiting pose for recognition $[17,8]$, the "pose" in our system is learned in a way that is directly tied to our end goal of action classification. 


\section{Pose Representation}

In this paper, we treat human pose as latent information and use it to assist the task of action recognition. Since we do not aim to obtain good pose estimation results in the end, the latent pose in our approach is not restricted to any specific type of pose representation. Because our focus is action recognition, we decide to choose a coarse exemplarbased pose representation. It is an action-specific variant of the "poselet" proposed in [2]. In this paper, we use the notation of "poselet" to refer to a set of patches not only with similar pose configuration, but also from the same action class. Fig. 3 illustrates the four poselets of a walking image. As we can see, the poselet normally covers more than one semantically meaningful part in terms of limbs and thus it is distinct from the background. So, the detection of poselets is more reliable than limb detection, especially with cluttered backgrounds.

In [2], a dataset is built where the joint positions of each human image are labeled in 3D space via a 2D-3D lifting procedure. We simply annotate the joint positions of human body in the 2D image space, as shown in Fig. 4. From the pose annotation, we can easily collect a set of patches with similar pose configuration. Based on the intuition that action-specific parts contain more discriminative information, we decide to select the poselets per action. For example, we would like to select a number of poselets from running-legs, or walking arms. The procedure of poselet selection for a particular action (e.g. running) is as follows: 1. We first divide the human pose annotation of the running images into four parts, legs, left-arm, right-arm, and upper-body; 2. We cluster the joints on each part into several clusters based their normalized $x$ and $y$ coordinates; 3 . We remove clusters with very few examples; 4 . Based on the pose clusters, we crop the corresponding patches from the images and form a set of poselets for the running action. Representative poselets from the running action are shown in Fig. 5. As we can see, among each poselet the appearance of each patch looks different, but they have very similar semantic meaning. As pointed in [2], this is also one advantage of using poselets. We repeat this process for other actions and obtain 90 poselets in total in the end.

In order to detect the presence of each poselet, we train a classifier for each poselet. We use the standard linear SVM and the histograms of Oriented Gradients feature proposed by Dalal and Triggs [3]. The positive examples are the patches from each poselet cluster. The negative examples are randomly selected from images which have the different action label to the positive examples. For example, when we train the classifier for one of "running-legs" poselets, we select the negative examples from all other action categories except for the running action. The learned running poselet templates are visualized in the last column in Fig. 5.

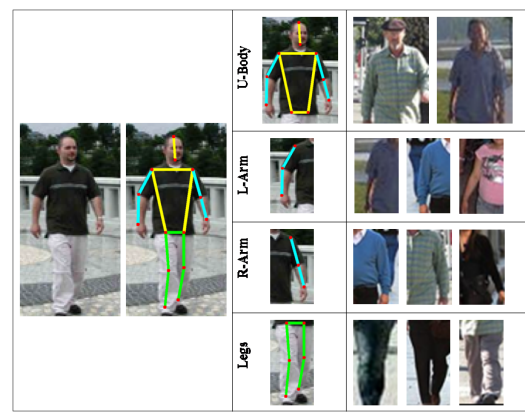

Figure 3. Visualization of the poselets for a walking image. Ground-truth skeleton is overlayed on image. Examples of poselets for each part are shown.

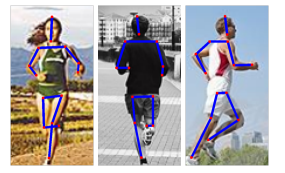

(a)

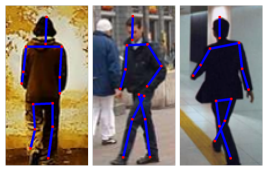

(b)

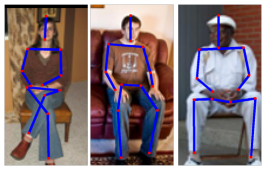

(d)

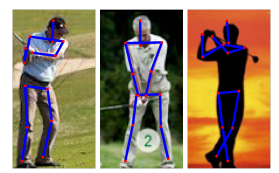

(c)

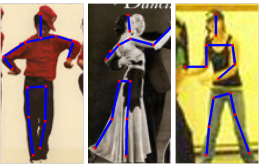

(e)
Figure 4. Sample images of the still image action dataset [11], and the ground truth pose annotation. The locations of 14 joints have been annotated on each action image. (a) Running; (b) Walking; (c) Playing Golf; (d) Sitting; (e) Dancing.

\section{Model Formulation}

Let $I$ be an image containing a person. In this paper, we consider a figure-centric representation where $I$ only contains one person centered in the middle of the image. This representation can be obtained from a standard pedestrian detection system. Let $Y$ be the action label of the person, and $L$ be the pose of the person. We denote $L$ as $L=\left(l_{0}, l_{1}, \ldots, l_{K-1}\right)$, where $K$ is the number of parts. In this paper, we choose $K=4$ corresponding to upper-body, legs, left-arm, and right-arm. The configuration of the $k$-th part $l_{k}$ is represented as $l_{k}=\left(x_{k}, y_{k}, z_{k}\right)$, where $\left(x_{k}, y_{k}\right)$ indicates the $(x, y)$ locations of the $k$-th part in the image, and $z_{k} \in \mathcal{Z}_{k}$ is the index of the chosen poselet for the $k$-th part. We have used $\mathcal{Z}_{k}$ to denote the poselet set corresponding to the part $k$. In this paper, we use $\left|\mathcal{Z}_{k}\right|$ as 26, 20, 20, 24 for the four parts: legs, left-arm, right-arm, and upper-body, based on our clustering results.

Similar to the standard pictorial structure models $[7,16]$ in human pose estimation, we use an undirected graph $\mathcal{G}=(\mathcal{V}, \mathcal{E})$ to constrain the configuration of the pose $L$. 


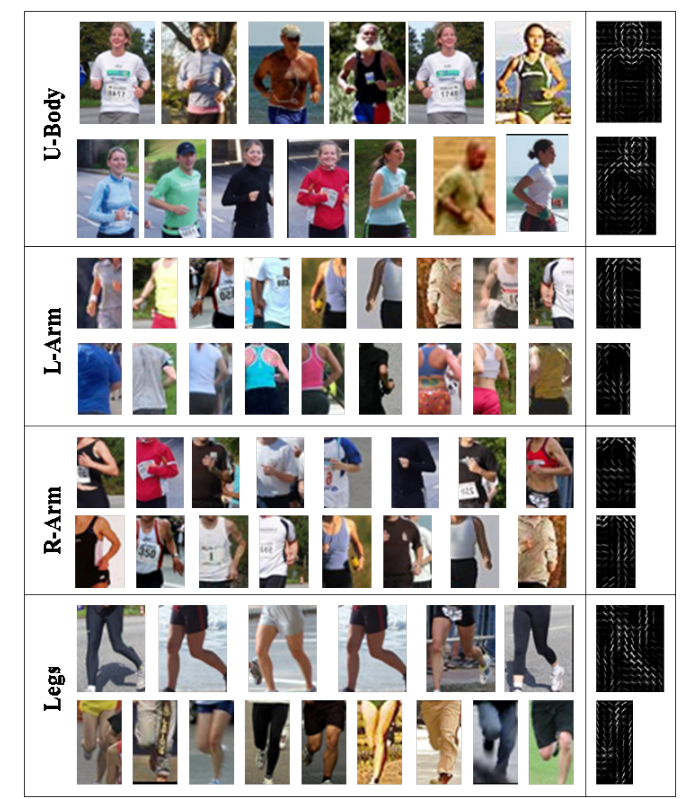

Figure 5. Examples of poselets for each part from the running action. Each row corresponds to one poselet. The last column is the visualization of the filters for each poselet learned from SVM + HOG.

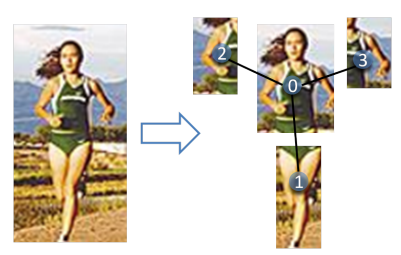

Figure 6. The four part star structured model. We divide the pose into four parts: legs, left-arm, right-arm, and upper-body.

Usually the kinematic tree of the human body is used. A vertex $j \in \mathcal{V}$ corresponds to the configuration $l_{j}$ of the $j$-th part, and an edge $(j, k) \in \mathcal{E}$ indicates the dependency between two connected parts $l_{j}$ and $l_{k}$. In this paper, we use a simple four part star structured model, as shown in Fig. 6. The upper-body part is the root node of $\mathcal{G}$ and other parts are connected to the root node. We emphasize that our algorithm is not limited to the four part star structure and can be easily generalized to other types of tree structures.

Our training data consists of images with ground-truth labels of their action classes and poses $(i . e .(x, y)$ location of each part and its chosen poselet). The ground-truth poselet of a part is obtained by tracing back the poselet cluster membership of this part. Given a set of $N$ training examples $\left\{\left(I^{(n)}, L^{(n)}, Y^{(n)}\right)\right\}_{n=1}^{N}$, our goal is to learn a model that can be used to assign the class label $Y$ to an unseen test image $I$. Note that during testing, we do not know the ground-truth pose $L$ of the test image $I$.

We are interested in learning a discriminative function $H: \mathcal{I} \times \mathcal{Y} \rightarrow \mathbb{R}$ over an image $I$ and its class label $Y$, where $H$ is parameterized by $\Theta$. During testing, we can predict the class label $Y^{*}$ of an input image $I$ as:

$$
Y^{*}=\arg \max _{Y \in \mathcal{Y}} H(I, Y ; \Theta)
$$

We assume $H(I, Y ; \Theta)$ takes the following form:

$$
H(I, Y ; \Theta)=\max _{L} \Theta^{T} \Psi(I, L, Y)
$$

where $\Psi(I, L, Y)$ is a feature vector depending on the image $I$, its pose configuration $L$ and its class label $Y$. We define $\Theta^{T} \Psi(I, L, Y)$ as follows:

$$
\begin{aligned}
& \Theta^{T} \Psi(I, L, Y)=\sum_{j \in \mathcal{V}} \alpha_{j}^{T} \phi\left(I, l_{j}, Y\right) \\
& +\sum_{(i, j) \in \mathcal{E}} \beta_{j k}^{T} \psi\left(l_{j}, l_{k}, Y\right)+\eta^{T} \omega\left(l_{0}, Y\right)+\gamma^{T} \varphi(I, Y)
\end{aligned}
$$

The model parameters $\Theta$ are simply the concatenation of the parameters in all the factors, i.e. $\Theta=\left\{\alpha_{j}: j \in\right.$ $\mathcal{V}\} \cup\left\{\beta_{j, k}:(j, k) \in \mathcal{E}\right\} \cup\{\gamma\}$. The details of the potential functions in Eqn. (3) are described below.

Part appearance potential $\alpha_{j}^{T} \phi\left(I, l_{j}, Y\right)$ : This potential function models the compatibility between the action class label $Y$, the configuration $l_{j}=\left(x_{j}, y_{j}, z_{j}\right)$ of the $j$-th part, and the appearance of the image patch extracted from the location $\left(x_{j}, y_{j}\right)$. It is parameterized as:

$\alpha_{j}^{T} \phi\left(I, l_{j}, Y\right)=\sum_{a \in \mathcal{Y}} \sum_{b \in \mathcal{Z}_{j}} \alpha_{j a b}^{T} \cdot \mathbb{1}_{a}(Y) \cdot \mathbb{1}_{b}\left(z_{j}\right) \cdot f\left(I\left(l_{j}\right)\right)$

where $\mathbb{1}_{a}(X)$ is an indicator that takes the value 1 if $X=$ $a$, and 0 otherwise. We use $f\left(I\left(l_{j}\right)\right)$ to denote the feature vector extracted from the patch defined by $l_{j}=\left(x_{j}, y_{j}, z_{j}\right)$ in the image $I$. The poselet set for the $j$-th part is denoted as $\mathcal{Z}_{j}$. The parameter $\alpha_{j a b}$ represents a template for the $j$-th part if the action label is $a$ and the chosen poselet for the $j$-th part is $b$.

Instead of keeping $f\left(I\left(l_{j}\right)\right)$ as a high dimensional vector, we simply use the output of a SVM classifier trained on a particular poselet as the single feature. We append a constant 1 to $f\left(I\left(l_{j}\right)\right)$ to learn a model with a bias term. In other words, let $f_{a b}\left(I\left(l_{j}\right)\right)$ be the score of the SVM trained with action $a$ and poselet $b$. Then the parameterization can be re-written as:

$$
\begin{aligned}
& \alpha_{j}^{T} \phi\left(I, l_{j}, Y\right)= \\
& \sum_{a \in \mathcal{Y}} \sum_{b \in \mathcal{Z}_{j}} \alpha_{j a b}^{T} \cdot \mathbb{1}_{a}(Y) \cdot \mathbb{1}_{b}\left(z_{j}\right) \cdot\left[f_{a b}\left(I\left(l_{j}\right)\right) ; 1\right]
\end{aligned}
$$

This trick greatly speeds up our learning algorithm. Similar tricks are used in [4]. 
Pairwise potential $\beta_{j k}^{T} \psi\left(l_{j}, l_{k}, Y\right)$ : This potential function represents the dependency between the $j$-th and the $k$ th part, for a given class label $Y$. Similar to [16], we use discrete binning to model the spatial relations between parts. We define this potential function as

$$
\beta_{j k}^{T} \psi\left(l_{j}, l_{k}, Y\right)=\sum_{a \in \mathcal{Y}} \beta_{j k a}^{T} \cdot \operatorname{bin}\left(l_{j}-l_{k}\right) \cdot \mathbb{1}_{a}(Y)
$$

where $\operatorname{bin}\left(l_{j}-l_{k}\right)$ is a feature vector that bins the relative location of the $j$-th part with respect to the $k$-th part according to the $(x, y)$ component of $l_{j}$ and $l_{k}$. Hence $\operatorname{bin}\left(l_{j}-l_{k}\right)$ is a sparse vector of all zeros with a single one for the occupied bin. Here $\beta_{j k a}$ is a model parameter that favors certain relative bins for the $j$-th part with respect to the $k$-th part for the action class label $a$.

Root location potential $\eta^{T} \omega\left(l_{0}, Y\right)$ : This potential function models the compatibility between the action class label $Y$ and the root location. Here $l_{0}$ denotes the configuration of the "root" part, i.e. upper-body in our case. It is parameterized as:

$$
\eta^{T} \omega\left(l_{0}, Y\right)=\sum_{a \in \mathcal{Y}} \eta_{a}^{T} \cdot \operatorname{bin}\left(l_{0}\right) \cdot \mathbb{1}_{a}(Y)
$$

We discretize the image grid into $h \times w$ spatial bins, and $\omega\left(l_{0}\right)$ is a length $h \times w$ sparse vector of all zeros with a single one for the spatial bin occupied by the root part. The parameter $\eta_{a}$ favors certain bins (possibly those in the middle of the image) for the location of the root part for the action label $a$. For example, for the running and walking actions, the root part may appear in the upper-middle part of the image with high probability, while for the sitting or playing golf action, the root part may appear in the centermiddle or lower-middle part of the image. This potential function deals with different root locations for different actions. It also allows us to handle the unreliability caused by the human detection system.

Global action potential $\gamma^{T} \varphi(I, Y)$ : This potential function represents a global template model for action recognition from still images without considering the pose configuration. It is parameterized as follows:

$$
\gamma^{T} \varphi(I, Y)=\sum_{a \in \mathcal{Y}} \gamma_{a}^{T} \cdot \mathbb{1}_{a}(Y) \cdot f(I)
$$

where $f(I)$ is a feature vector extracted from the whole image $I$. The parameter $\gamma_{a}$ is a template for the action class $a$. This potential function measures the compatibility between the model parameter $\gamma$ and the combination of image observation $f(I)$ and its class label $Y$. Similar to the part appearance model, we represent $f(I)$ as a vector of outputs of a multi-class SVM classifier.

\section{Learning and Inference}

We now describe how to infer the action label $Y$ given the model parameters $\Theta$ (Sec. 4.1), and how to learn the model parameters from a set of training data (Sec. 4.2)

\subsection{Inference}

Given the model parameters and a test image $I$, we can enumerate all the possible action labels $Y \in \mathcal{Y}$ and predict the action label $Y^{*}$ of $I$ according to Eqn. (1). For a fixed $Y$, we need to solve an inference problem of finding the best pose $L^{\text {best }}$ as follows:

$$
\begin{aligned}
& L^{\text {best }}=\arg \max _{L} \Theta^{T} \Psi(I, L, Y) \\
& =\arg \max _{L}\left(\sum_{j \in \mathcal{V}} \alpha_{j}^{T} \phi\left(I, l_{j}, Y\right)+\sum_{(i, j) \in \mathcal{E}} \beta_{j k}^{T} \psi\left(l_{j}, l_{k}, Y\right)\right. \\
& \left.\quad+\eta^{T} \omega\left(l_{0}, Y\right)\right)
\end{aligned}
$$

Note for a fixed $Y$, the global action potential function is a constant and has nothing to do with the pose $L$, so we omit it from above equation. Since we assume a star model on $L$, the inference problem in Eqn. (9) can be efficiently solved via dynamic programming.

In this paper, we choose the size of relative location binning $\operatorname{bin}\left(l_{j}-l_{k}\right)$ as $32 \times 15$. With such a discrete binning scheme, the inference can be directly solved by dynamic programming efficiently even without using the generalized distance transform [7]. The inference for a fixed $Y$ on an image only takes $0.015 s$ with our MATLAB/MEX implementation.

\subsection{Learning}

Now we describe how to train the model parameters $\Theta$ from $N$ training examples $\left\{I^{n}, L^{n}, Y^{n}\right\}_{n=1,2, \ldots, N}$. If we assume the pose $L$ is unobserved on the training data, we can learn $\Theta$ using the latent SVM formulation $[6,21]$ as follows:

$$
\begin{array}{ll}
\min _{\Theta, \xi^{n} \geq 0} & \Theta^{T} \Theta+C \sum_{n} \xi^{n} \\
\text { s.t. } & \underbrace{\max _{L} \Theta^{T} \Psi\left(I^{n}, L, Y^{n}\right)}_{H\left(I^{n}, Y^{n} ; \Theta\right)}-\underbrace{\max _{L} \Theta^{\top} \Psi\left(I^{n}, L, Y\right)}_{H\left(I^{n}, Y ; \Theta\right)} \\
& \geq \Delta\left(Y, Y^{n}\right)-\xi^{n}, \quad \forall n, \quad \forall Y \in \mathcal{Y}
\end{array}
$$

where $\Delta\left(Y, Y^{n}\right)$ is a function measuring the loss incurred by classifying the example $I^{n}$ to be $Y$, while the true class label is $Y^{n}$. We use the 0-1 loss defined as follows:

$$
\Delta\left(Y, Y^{n}\right)= \begin{cases}1 & \text { if } Y \neq Y^{n} \\ 0 & \text { otherwise }\end{cases}
$$

The constraint in Eqn. (10) specifies the following intuition. For the $n$-th training example, we want the score 
$H\left(I^{n}, Y ; \Theta\right)=\arg \max _{L} \Theta^{T} \Psi\left(I^{n}, L, Y\right)$ to be high when $Y$ is the true class label, i.e. $Y=Y^{n}$. In particular, we want the score $H\left(I^{n}, Y^{n} ; \Theta\right)$ to be higher than the score associated with any hypothesized class label $H\left(I^{n}, Y ; \Theta\right)$ by 1 if $Y \neq Y^{n}$.

Now since $L$ is observed on training data, one possible way to learn $\Theta$ is to plug-in the ground-truth pose in Eqn. (10), i.e. optimize the following problem:

$$
\begin{array}{ll}
\min _{\Theta, \xi^{n} \geq 0} & \Theta^{T} \Theta+C \sum_{n} \xi^{n} \\
\text { s.t. } & \Theta^{T} \Psi\left(I^{n}, L^{n}, Y^{n}\right)-\max _{L} \Theta^{\top} \Psi\left(I^{n}, L, Y\right) \\
& \geq \Delta\left(Y, Y^{n}\right)-\xi^{n}, \forall n, \forall Y \in \mathcal{Y}
\end{array}
$$

Our initial attempt of using Eqn. (12) suggests it does not perform as well as Eqn. (10). We believe it is because the learning objective in Eqn. (12) assumes that we will have access to the correct pose estimation at run-time. This is unrealistic. On the other hand, the learning objective in Eqn. (10) mimics the situation at run-time, when we are faced with a new image without the ground-truth pose. So we will use the formulation in Eqn. (10) from now on. But we would like to point out that Eqn. (10) does not ignore the ground-truth pose information on the training data. That information has been implicitly built into the features which are represented as outputs of SVM classifiers. Those SVM classifiers are learned using the ground-truth information of the pose on the training data.

The training problem in Eqn. (10) can be solved by the non-convex cutting plane algorithm in [5], which is an extension of the popular convex cutting plane algorithm [12] for learning structural SVM [1]. We briefly outline the algorithm here.

Consider the following unconstrained formulation which is equivalent to Eqn. (10):

$$
\begin{aligned}
& \Theta=\arg \min _{\Theta} \Theta^{T} \Theta+C \sum_{n} R_{n}(\Theta) \quad \text { where } \\
& R_{n}(\Theta)=\max _{Y}\left(\Delta\left(Y, Y^{n}\right)+H\left(I^{n}, Y ; \Theta\right)\right) \\
& -H\left(I^{n}, Y^{n} ; \Theta\right)
\end{aligned}
$$

In a nutshell, the learning algorithm in [5] iteratively builds an increasingly accurate piecewise quadratic approximation of Eqn. (13) based on the subgradient $\partial_{\Theta}\left(\sum_{n} R_{n}(\Theta)\right)$. It can be shown that the subgradient $\partial_{\Theta}\left(\sum_{n} R_{n}(\Theta)\right)$ is related to the most-violated constraint of Eqn. (10). So in essence, the algorithm iteratively adds the most-violated constraint of Eqn. (10) and solves a piecewise quadratic approximation at each iteration. It has been proved that only a small number of constraints are needed in order to achieve an reasonably accurate approximation to the original problem [1].
Now the key issue is how to compute the subgradient $\partial_{\Theta}\left(\sum_{n} R_{n}(\Theta)\right)$. Since

$$
\partial_{\Theta}\left(\sum_{n} R_{n}(\Theta)\right)=\sum_{n} \partial_{\Theta} R_{n}(\Theta)
$$

all we need to do it to figure out how to compute $\partial_{\Theta} R_{n}(\Theta)$. Let us define:

$$
\begin{aligned}
& \left(Y^{*}, L^{*}\right)=\arg \max _{Y, L} \Delta\left(Y, Y^{n}\right)+\Theta^{T} \Psi\left(I^{n}, L, Y\right) \\
& \widehat{L}=\arg \max _{L} \Theta^{T} \Psi\left(I^{n}, L, Y^{n}\right)
\end{aligned}
$$

It can be shown that $\partial_{\Theta} R_{n}(\Theta)$ can be calculated as

$$
\partial_{\Theta} R_{n}(\Theta)=\Psi\left(I^{n}, L^{*}, Y^{*}\right)-\Psi\left(I^{n}, \widehat{L}, Y^{n}\right)
$$

The calculation of the subgradient $\partial_{\Theta} R_{n}(\Theta)$ involves solving two inference problems in Eqn. $(15,16)$. As mention in Sec. 4.1, the inference on $\arg \max _{L}$ can be efficiently solved via dynamic programming. The inference on $\arg \max _{Y}$ is easy too, since the number of possible choices of $Y$ is small (e.g. $|\mathcal{Y}|=5$ in our case). So we can simply enumerate all possible $Y \in \mathcal{Y}$.

\section{Experiments}

We first test our algorithm on the still image action dataset collected by Ikizler-Cinbis et al. [11]. This dataset consists of still images from five action categories: running, walking, playing golf, sitting, and dancing. The images of this dataset are downloaded from the Internet. So there are a lot of pose variations and cluttered backgrounds in the dataset. In total, there are 2458 images in the dataset. We further increase the size and pose variability of the dataset by mirror-flipping all the images. Most of actions in the dataset do not have axial symmetry. For example, runningto-left and running-to-right appear very different in the image. So mirror-flipping makes the dataset more diverse. We manually annotate the pose with 14 joints on the human body on all the images in the dataset, as shown in Fig. $4^{1}$. We select $1 / 3$ of the images from each action category to form the training set, and the rest of the images are used for testing. We also ensure the testing set does not contain the mirror-flipped version of any training image. Since we focus on action classification rather than human detection, we simply normalize each image into the same size and put the human figure in the center of the image, based on the pose annotation information.

At the training stage, we create 90 poselets in total from the training set following the method described in Section 2. For each poselet, we train an SVM classifier based on the HOG descriptors extracted from image patches at

\footnotetext{
${ }^{1}$ The annotations are available online (http://www.sfu.ca/ wya16/latent/latentpose.html)
} 


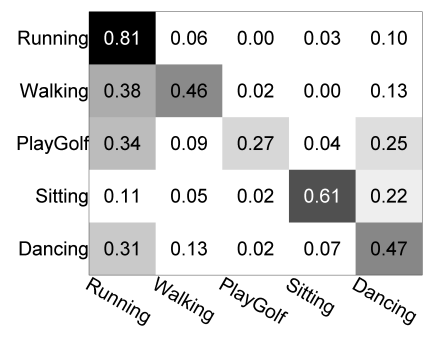

(a)

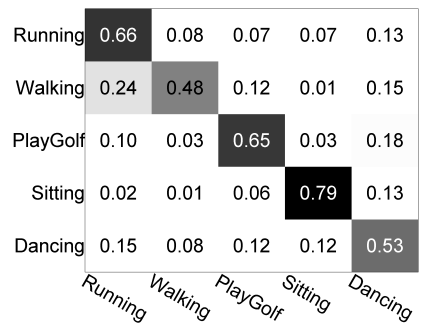

(b)

Figure 7. Confusion matrices of the classification results on the still image action dataset: (a) baseline (b) our approach. Horizontal rows are ground truths, and vertical columns are predictions.

the ground-truth locations of the corresponding poselet in the training set. Examples of trained templates for running poselets are visualized in the last column of Fig. 5. We compare our approach with a multi-classification SVM with HOG descriptors as the baseline. Note that the outputs of this baseline are also used to model the global action potential function in our approach. The confusion matrices of the baseline and our method on the testing set are shown in Fig. 7 (a),(b). Table 1 summarizes the comparison between our result and the baseline. Since the testing set is imbalanced, e.g. the number of running examples are more than twice of the playing-golf and sitting examples, we report both overall and mean per class accuracies. For both overall and mean per class accuracies, our method outperforms the baseline.

We also apply our trained model on a Youtube video dataset originally collected by Niebles et al. $[14]^{2}$. IkizlerCinbis et al. [11] have annotated 11 videos of this dataset. The action of each human figure on each frame has been annotated by one of the five action categories. The bounding box information of the human figure returned by a standard human detection algorithm is also provided by IkizlerCinbis et al. [11]. In total, there are 777 human figures. We normalize each human figure into the same size based on the bounding box information and then run our model, which is trained from the still image dataset. To show the generalization power of our method, we use exactly the same model learned from our previous experiment on the still image action dataset without any re-training on the Youtube dataset. The confusion matrix of our method is given in Fig. 8. Ta-

\footnotetext{
${ }^{2}$ http://vision.stanford.edu/projects/extractingPeople.html
}

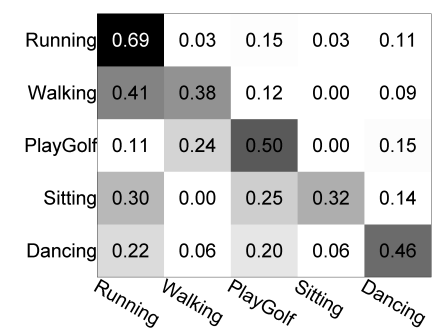

Figure 8. Confusion matrix of the classification results of our approach on the Youtube dataset. Horizontal rows are ground truths, and vertical columns are predictions.

\begin{tabular}{|l||c|c|}
\hline method & overall & mean per-class \\
\hline \hline Baseline & 56.45 & 52.46 \\
Our approach & $\mathbf{6 1 . 0 7}$ & $\mathbf{6 2 . 0 9}$ \\
\hline
\end{tabular}

Table 1. Results on the still image action dataset. We report both overall and mean per class accuracies due to the class imbalance.

\begin{tabular}{|l||c|c|}
\hline method & overall & mean per-class \\
\hline \hline Baseline & 46.98 & 40.52 \\
Our approach & $\mathbf{5 0 . 5 8}$ & $\mathbf{4 6 . 7 3}$ \\
\hline \hline$[11]$ (MultiSVM) & 59.35 & N/A \\
[11] (Best) & 63.61 & N/A \\
\hline
\end{tabular}

Table 2. Results on the Youtube dataset. We report both overall and mean per class accuracies due to the class imbalance.

ble 2 shows the comparison of our method with the baseline SVM classifier on HOG features trained from the same training set. Our method performs much better in terms of both overall and mean per-class accuracies. Our results are lower than the best results without temporal smoothing reported in [11]. This is likely because the method in [11] uses an additional step of perturbing the bounding box on the training set to account for the errors of human localization. If we use the same trick in our method, the performance will probably improve as well.

Visualization of Latent Pose: We can also visualize the latent poses learned by our model. But first, we need to point out that our model is trained for action classification, not pose estimation. We simply treat the pose as latent information in the model that can help solve the action classification task. Since our model is not directly optimized for pose estimation, we do not expect to get pose estimation results that are "good" in the usual sense. When measuring the performance of a pose estimation, people typically examine how closely the localized body parts (torso, arm, legs, etc.) match the ground-truth locations of the parts in the image. But since our final goal is action classification, we are not aiming to correctly localize the body parts, but rather focus on localizing the body parts that are useful for action classification.

Fig. 9 shows the visualization of the latent poses superimposed on the original images. For the $k$-th part with $l_{k}=\left(x_{k}, y_{k}, z_{k}\right)$, we place the chosen poselet $z_{k}$ at the 


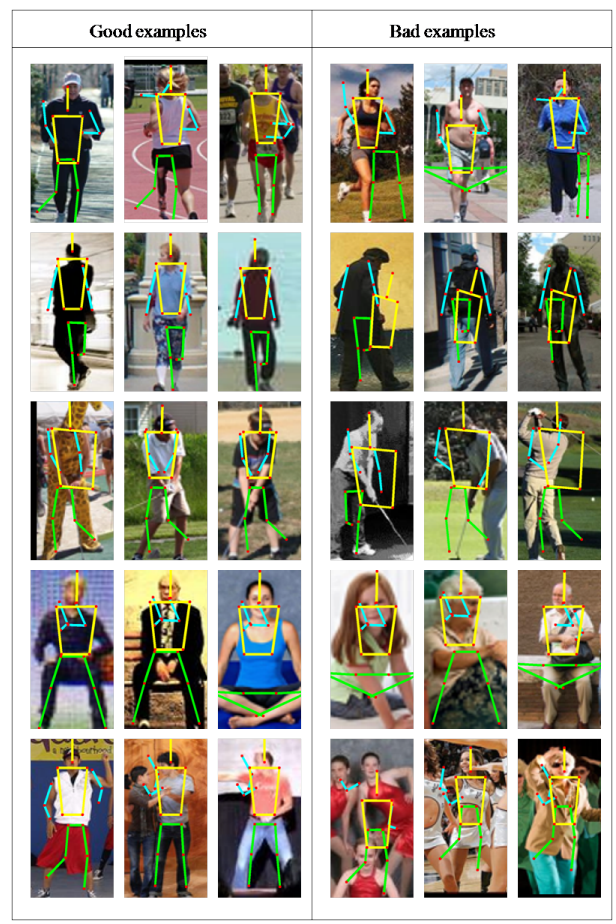

Figure 9. Example visualizations of the latent poses on test images. For each action, we manually select some good estimation examples and bad examples. The action for each row (from top) is running, walking, palying golf, sitting and dancing respectively.

location $\left(x_{k}, y_{k}\right)$ in the image. The skeleton used for a particular poselet is obtained from the cluster center of the joint locations of the corresponding poselet. In terms of pose estimation in the usual sense, those results are not accurate. However, we can make several interesting observations. In the "sitting" action, our model almost always correctly localizes the legs. In particular, it mostly chooses the poselet that corresponds to the "A" shaped-legs (e.g. first two images in the fourth row) or the triangle-shaped legs (e.g. the third image in the fourth row). It turns out the legs of a person are extremely distinctive for the "sitting" action. So our model "learns" to focus on localizing the legs for the sitting action, in particular, our model learns that the "A" shapedlegs and the triangle-shaped legs are most discriminative for the sitting action. For the sitting action, the localized arms are far from their correct locations. From the standard pose estimation point of view, this is considered as a failure case. But for our application, this is fine since we are not aiming to correctly localize all the parts. Our model will learn not to use the localizations of the arms to recognize the sitting action. Another example is the "walking" action (the images in the second row). For this action, our model almost always correctly localizes the arms hanging on the two sides of the torso, even on the bad examples. This is because "hanging arms" is a very distinctive poselet for the walking action. So our model learns to focus on this particular part for walking, without getting distracted by other parts.

\section{Conclusion}

We have presented a model that integrates action recognition and pose estimation. The main novelty of our model is that although we consider these two problems together, our end goal is action recognition, and we treat the pose information as latent variables in the model. The pose is directly learned in a way that is tied to action recognition. This is very different from other work that learns a pose estimation system separately, then uses the output of the pose estimation to train an action recognition system. Our experimental results demonstrate that by inferring the latent pose, we can improve the final action recognition results.

\section{References}

[1] Y. Altun, T. Hofmann, and I. Tsochantaridis. SVM learning for interdependent and structured output spaces. In Machine Learning with Structured Outputs. MIT Press, 2006.

[2] L. Bourdev and J. Malik. Poselets: Body part detectors training using 3d human pose annotations. In ICCV, 2009.

[3] N. Dalal and B. Triggs. Histogram of oriented gradients for human detection. In CVPR, 2005.

[4] C. Desai, D. Ramanan, and C. Fowlkes. Discriminative models for multi-class object layout. In $I C C V, 2009$.

[5] T.-M.-T. Do and T. Artieres. Large margin training for hidden markov models with partially observed states. In ICML, 2009.

[6] P. Felzenszwalb, D. McAllester, and D. Ramanan. A discriminatively trained, multiscale, deformable part model. In CVPR, 2008.

[7] P. F. Felzenszwalb and D. P. Huttenlocher. Pictorial structures for object recognition. IJCV, 61(1):55-79, January 2005.

[8] V. Ferrari, M. Marín-Jiménez, and A. Zisserman. Pose search: retrieving people using their pose. In CVPR, 2009.

[9] D. A. Forsyth, O. Arikan, L. Ikemoto, J. O'Brien, and D. Ramanan. Computational studies of human motion: Part 1, tracking and motion synthesis. Foundations and Trends in Computer Graphics and Vision, 1(2/3):77-254, July 2006.

[10] N. Ikizler, R. G. Cinbis, S. Pehlivan, and P. Duygulu. Recognizing actions from still images. In ICPR, 2008.

[11] N. Ikizler-Cinbis, R. G. Cinbis, and S. Sclaroff. Learning actions from the web. In ICCV, 2009.

[12] T. Joachims, T. Finley, and C.-N. Yu. Cutting-plane training of structural SVMs. Machine Learning, 2008.

[13] I. Laptev, M. Marszalek, C. Schmid, and B. Rozenfeld. Learning realistic human actions from movies. In CVPR, 2008.

[14] J. C. Niebles, B. Han, A. Ferencz, and L. Fei-Fei. Extracting moving people from internet videos. In ECCV, 2008.

[15] J. C. Niebles, H. Wang, and L. Fei-Fei. Unsupervised learning of human action categories using spatial-temporal words. In $B M V C$, volume 3, pages 1249-1258, 2006.

[16] D. Ramanan. Learning to parse images of articulated bodies. In NIPS, volume 19, pages 1129-1136, 2007.

[17] D. Ramanan and D. A. Forsyth. Automatic annotation of everyday movements. In NIPS. MIT Press, 2003.

[18] C. Schuldt, I. Laptev, and B. Caputo. Recognizing human actions: a local SVM approach. In ICPR, volume 3, pages 32-36, 2004.

[19] C. Thurau and V. Hlaváč. Pose primitive based human action recognition in videos or still images. In CVPR, 2008.

[20] Y. Wang, H. Jiang, M. S. Drew, Z.-N. Li, and G. Mori. Unsupervised discovery of action classes. In $C V P R, 2006$.

[21] Y. Wang and G. Mori. Max-margin hidden conditional random fields for human action recognition. In CVPR, 2009. 